\title{
A machine learning-based framework for diagnosis of COVID-19 from chest X-ray images
}

\author{
Jawad Rasheed $^{1} \mathbb{D} \cdot$ Alaa Ali Hameed ${ }^{1} \cdot$ Chawki Djeddi $^{2} \cdot$ Akhtar Jamil $^{1} \cdot$ Fadi Al-Turjman $^{3}$
}

Received: 12 September 2020 / Revised: 5 November 2020 / Accepted: 20 November 2020 / Published online: 2 January 2021

(c) International Association of Scientists in the Interdisciplinary Areas 2021

\begin{abstract}
Corona virus disease (COVID-19) acknowledged as a pandemic by the WHO and mankind all over the world is vulnerable to this virus. Alternative tools are needed that can help in diagnosis of the coronavirus. Researchers of this article investigated the potential of machine learning methods for automatic diagnosis of corona virus with high accuracy from X-ray images. Two most commonly used classifiers were selected: logistic regression (LR) and convolutional neural networks (CNN). The main reason was to make the system fast and efficient. Moreover, a dimensionality reduction approach was also investigated based on principal component analysis (PCA) to further speed up the learning process and improve the classification accuracy by selecting the highly discriminate features. The deep learning-based methods demand large amount of training samples compared to conventional approaches, yet adequate amount of labelled training samples was not available for COVID-19 $\mathrm{X}$-ray images. Therefore, data augmentation technique using generative adversarial network (GAN) was employed to further increase the training samples and reduce the overfitting problem. We used the online available dataset and incorporated GAN to have 500 X-ray images in total for this study. Both CNN and LR showed encouraging results for COVID-19 patient identification. The LR and CNN models showed 95.2-97.6\% overall accuracy without PCA and 97.6-100\% with PCA for positive cases identification, respectively.
\end{abstract}

Keywords Artificial neural network · Computer-aided diagnosis · COVID-19 · Image classification · Principal component analysis

\section{Introduction}

The COVID-19, an infectious virus, emerged in China at the end of December 2019. It has spread to at least 213 countries around the globe as of August 02, 2020. Therefore, on March 11, 2020, the WHO declared the COVID-19 outbreak as pandemic. According to Johns Hopkins University, ${ }^{1}$ the tally of confirmed cases has exceeded 25,225,566 while the death toll is over 847,676 until August 30, 2020. It is also confirmed that $17,688,088$ people diagnosed with COVID19 have recovered from the disease so far.

Jawad Rasheed

jawadrasheed@ieee.org

1 Department of Computer Engineering, Istanbul Sabahattin Zaim University, 34303 Istanbul, Turkey

2 Department of Mathematics and Computer Science, Larbi Tebessi University, 12018 Tébessa, Algeria

3 Artificial Intelligence Department, Research Center for AI and IoT, Near East University, Nicosia, Mersin 10, Turkey
Besides affecting humans, this huge family of viruses, called coronaviruses, mostly affects various animals and other species such as bats, cats, and camels. These animal coronaviruses once infect a human, can then become a cause of horizontal transfer (human-to-human) such as with severe acute respiratory syndrome coronavirus (SARS-CoV), Middle East respiratory syndrome coronavirus (MERS-CoV), and currently with this infectious virus named as COVID-19 (also known as SARS-CoV-2). According to National Institutes of Health, ${ }^{2}$ the SARS-CoV, MERS-CoV, and SARS$\mathrm{CoV}-2$ viruses are originated in animal reservoir. The history of human coronaviruses started in 1965 when B814 virus was discovered in a human body by [1]. In last 20 years, scientists have discovered more than five new human coronaviruses that have caused substantial rise in mortality and morbidity [2].

\footnotetext{
1 Johns Hopkins University: COVID-19 Maps-John Hopkins Coronavirus Research Center, https://coronavirus.jhu.edu/map.html

2 National Institutes of Health: https://www.nih.gov/health-infor mation/coronavirus
} 
Until now, scientists could not find any treatment for COVID-19. The symptoms of the virus involve respiratory illness of mild to severe intensity, sore throat, coughing, diarrhoea etc. [3]. This virus spreads in the form of droplets of saliva when someone sneezes or through a physical contact [4]. However, recently, WHO declared that it is also airborne. On the other hand, elder people or humans with chronic medical history or critical diseases like cancer, chronic respiratory problem, cardiovascular disease or diabetes may require special treatment. As mentioned by WHO, ${ }^{3}$ such people are more vulnerable and develop serious illness when contracted with COVID-19. So far, the only response to this virus is to rely on isolation, quarantine, and infection-control measures to combat and control the COVID-19 outbreak [5].

Early detection of this disease can help in timely isolation of patients and monitor their health status [6]. Machine learning-based approaches can be used that analyze the lungs' X-ray/CT images to identify patients affected by pneumonia due to COVID-19 infection. This technique can be used as an alternative where COVID-19 kits are not available, especially in developing countries where a large population is affected by this virus but no measures could be arranged to confirm the suspects for COVID-19.

Supervised learning techniques have shown great progress for early detection and diagnosis of diseases. For instance, in [7], a decision support system is proposed for prediction of diabetes using machine learning techniques. Three machine-learning algorithms were used that includes CNN, random forest, and support vector machine (SVM). The experiments showed promising results for classification of 768 patients into diabetic and non-diabetic groups. Similarly, [8] compared most popular machine learning techniques commonly used for detection of breast cancer. The methods implemented include random forest, k-nearestneighbor ( $\mathrm{kNN})$ and Naïve Bayes classifiers. In [9], a wavelet transforms and SVM-based methodology is proposed for categorization of brain tumors into two different classes as benign or malignant. Sun et al. [10] measured the diagnostic performance by investigating 15 different classification and several feature selection methods in glioma grading. The results indicated that the combination of feature selection with linear SVM and multilayer perceptron (MLPC) achieved the best performance. A comprehensive assessment of machine learning-based futuristic approaches in medical image analysis can be found in [11].

In spite the success of machine learning approaches, their performance is highly affected by the quality of the handengineered features. The hand-engineered features are not optimal and also a time-consuming task [12]. This is the

\footnotetext{
${ }^{3}$ WHO: World Health Organization, https://www.who.int/
}

main drawback of machine learning approach, despite the success, these techniques suffer from serious degradation in performance. The alternative approach, which has become hot research topic in recent era, is to extract automatic optimal features from the input data. These techniques have not only removed the barrier of manual feature extraction but also improved the classification accuracy.

Several techniques based on deep learning (DL) have been put forward to achieve the above goals: automatic feature extraction and improving classification accuracy. With improvements in computer hardware, it has become feasible to train more and more complex models. DL approaches, especially CNNs have shown their efficiency for various computer vision tasks such as object detection, natural language processing, image segmentation and classification. Motivated from the computer vision community, the medical community has also adopted the model to solve many medical image analysis tasks. For instance, in [13], authors investigated two well-known deep neural networks: Inception and VGG16, for diagnosis of pneumonia from images of chest $\mathrm{X}$-ray. It was reported that VGG16 resulted in higher classification accuracy compared to Inception model. Segmentation of lung X-ray images using deep CNNs is proposed in [14] for improving the performance during clinical diagnosis of various diseases in lungs such as lung cancer, tuberculosis, or lung opacities. Van Tulder and De Bruijne [15] used an unsupervised feature learning based on restricted Boltzmann machines for feature extraction with a generative learning objectives. It combines both generative and discriminative learning objectives with convolutional classification for categorization of lung computed tomography (CT) images. Based on histology images, [16] developed a reliable system to enhance the diagnostic quality for identification of breast cancer. Particularly, two machine learning approaches were compared: SVM was used as classifier using handcrafted features while another approach is based on CNNs for automatic feature learning and classification. The results indicated that $\mathrm{CNN}$ performed well than the classifier based on handcrafted features.

In [17], various $\mathrm{CNN}$ architectures were investigated for classification and detection of interstitial lung disease and thoraco-abdominal lymph node, respectively. Further analysis was performed to evaluate the effect of spatial image context and dataset scale on classifier performance, and the application of transfer learning from pre-trained ImageNet in the domain of image analysis.

Besides conventional down sampling layers, [18] proposed an atrous convolution as an alternative layer in the deep CNNs. Since CNNs mostly exploit down sampling layers to increase the receptive field and gain abstract semantic information; however, the down sampling also decreases the feature maps' spatial dimensions that may not be desirable for semantic segmentation tasks. The atrous convolutions, 
on the other hand, can increase the receptive field without changing the feature maps' spatial dimension. A comprehensive analysis of the present day furtherance in machine learning, specifically in DL that helped in distinguishing, categorizing and gauging the patterns in medical images is presented in [19].

Despite the success of DL models, their application for the examination of medical image is particularly a challenging task. The deeper networks usually require large datasets for training; however, large labeled datasets of medical images are not abundant compared to vision related datasets such as ImageNet [20]. In addition, the imbalance datasets and poor representation can make the problem even more complex. Moreover, the privacy and confidentiality concerns related to medical data of patients also limit the access to the data [21].

To overcome this limited availability of labelled data, researchers proposed data augmentation techniques. Data augmentation technique is utilized to elevate the amount of training data by working in various transformation such as rotation, scaling, translations etc. on the original data. It also helps reducing the common problem of overfitting. A detailed review of recent augmentation techniques for DL are described in details in [22].

Data augmentation is also adopted in various medical image analysis applications such as semantically segmenting the various sclerosis lesions using magnetic resonance imaging (MRI) of brain [23], cardiac image enhancement and segmentation or reconstruction [24, 25], mitosis detection in breast cancer histology images [26] and brain tumor segmentation using MRI images [27].

Conversely, researchers have also taken advantage of AIbased internet of things (IoT) in the field of medical sciences for diagnostic purposes. Such as, [28] proposed an application of IoT that detects respiratory motion at real time by monitoring diabetic patient's breathing to diagnose diabetic ketoacidosis (DKA). They used C-band sensing method by exploiting microwave-sensing platform (MSP) as a nonintrusive respiratory monitoring system. They further used peak detection algorithm that acquires respiratory rate for identification of Kussmaul breathing. Similarly, [29] used S-band sensing technique to characterize wandering patterns in patients suffering from dementia. Later, researchers incorporated SVM as a pattern classification algorithm.

In this article, researchers propose a machine learningbased framework for detection of COVID-19 from X-ray images. We used one traditional machine learning approach, LR, which is an efficient and simple method to implement. In addition, for DL, we implemented deep CNN. Moreover, we investigated the application of feature selection technique (PCA) to lower the computational time and enhance the overall accuracy.
The rest of the paper is organized as follows. Section 2 summarizes the details of dataset used in this study and the proposed methodology. Section 3 outlines the experimental setup and presents the results, while Sect. 4 provides the summary and discussion. Finally, the paper concludes at Sect. 5.

\section{Materials and methods}

This section defines the dataset and methodologies used for prediction of COVID-19 virus. It outlines the experimental dataset, data augmentation technique, feature selection method, DL and machine learning predictive models incorporated in this work. Workflow of system put forward by researchers is shown in Fig. 1, that depicts the preprocessing performed on compiled dataset and various sets of features pertaining high information are extracted via PCA. These extracted sets of features are then fed to proposed DL model and traditional machine learning network for training, and later their performances are evaluated on respective test sets.

\subsection{Dataset}

The experimental work is performed by acquiring X-rays images of COVID-19 infected persons from COVID-19 images data collection provided by Joseph Paul Cohen, ${ }^{4}$ and normal X-rays images from Chest X-ray Images ${ }^{5}$ (pneumonia) repository as healthy individuals. At the time of this study, COVID-19 images data collection contains only 198 X-rays images pertaining to COVID-19 affected cases. The experimental work is developed with the help of X-ray images of sufferers from COVID-19 data collection by taking $198 \mathrm{X}$-rays images of virus-affected patients and $210 \mathrm{X}$-rays images of healthy individuals from Chest $\mathrm{X}$-ray Images (pneumonia) repository. The original size of the compiled dataset varies in range of $1112 \times 624$ to $2170 \times 1953$ pixels. For this experimental work, all images are scaled to $512 \times 512$ pixels. Table 1 summarizes the data set used in this study. The Fig. 2 depicts few samples from both classes of dataset. Data augmentation was applied to add additional data to create class balance. The final version of dataset contains 250 samples for each class. The class label 1 was used to represent COVID-19 positive cases while 0 represents normal healthy cases.

\footnotetext{
4 Joseph Paul Cohen: COVID-19 image data collection, https://doi. org/2003.11597 (last accessed 2020/07).

${ }^{5} \mathrm{P}$. Mooney: Chest X-Ray Images (Pneumonia), https://doi. org/10.17632/rscbjbr9sj.2 https://www.kaggle.com/paultimothymoon ey/chest-xray-pneumonia (last accessed 2020/07).
} 


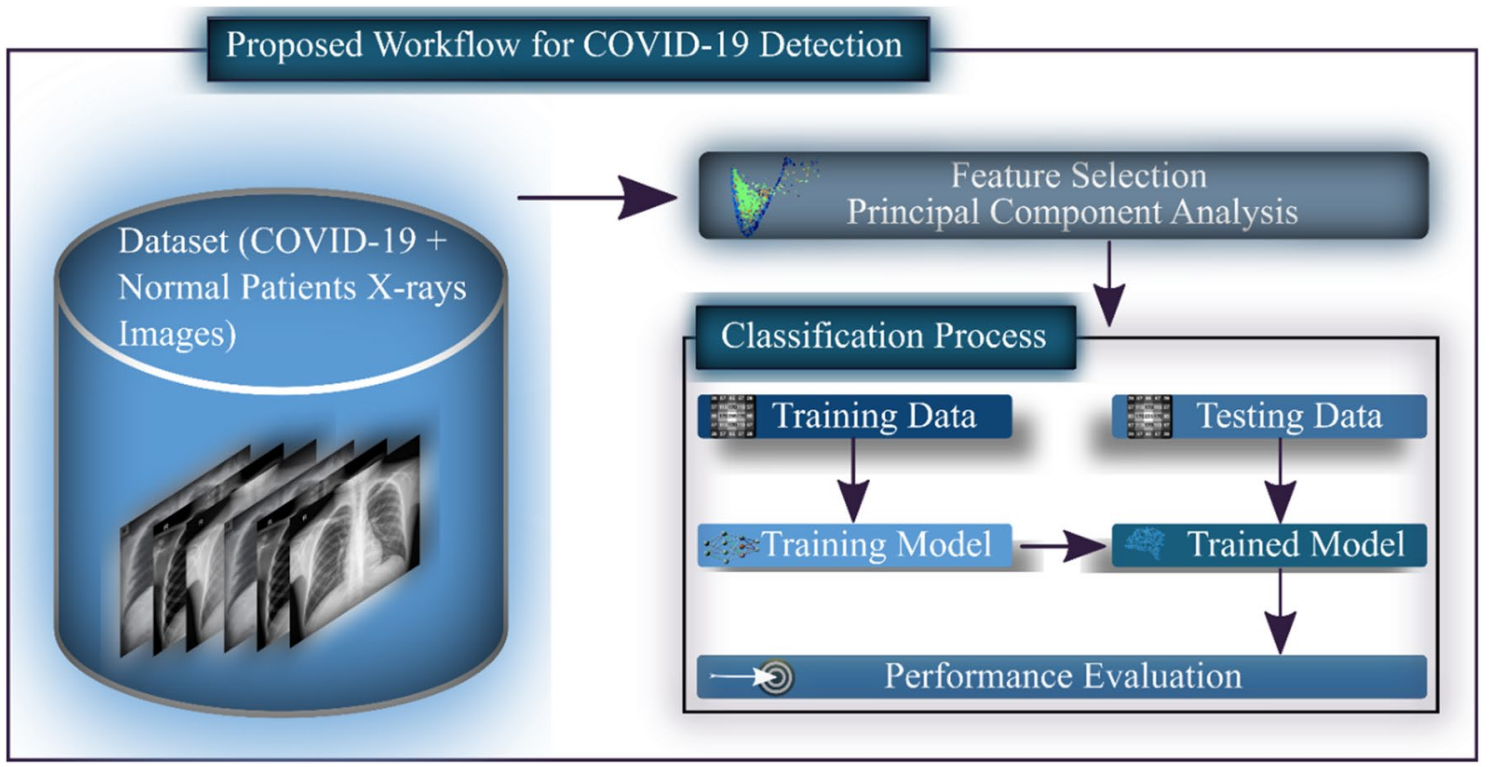

Fig. 1 Workflow of proposed system

Table 1 Dataset information

\begin{tabular}{llll}
\hline Cases & $\begin{array}{l}\text { Original sam- } \\
\text { ples }\end{array}$ & $\begin{array}{l}\text { Augmented } \\
\text { samples }\end{array}$ & Total \\
\hline COVID-19 & 198 & 52 & 250 \\
Healthy individuals & 210 & 40 & 250 \\
Total & 408 & 92 & 500 \\
\hline
\end{tabular}

\subsection{Data augmentation with GAN}

GAN has been extensively utilized in numerous image generation functions that produces artificial data-like real data. This can help to overcome the issue of small number of training samples thus eliminates class imbalance problem. The GAN architecture consists of multilayer perceptron which has two main elements: generator $(\mathrm{G})$ and discriminator (D) [30]. These two components compete with each
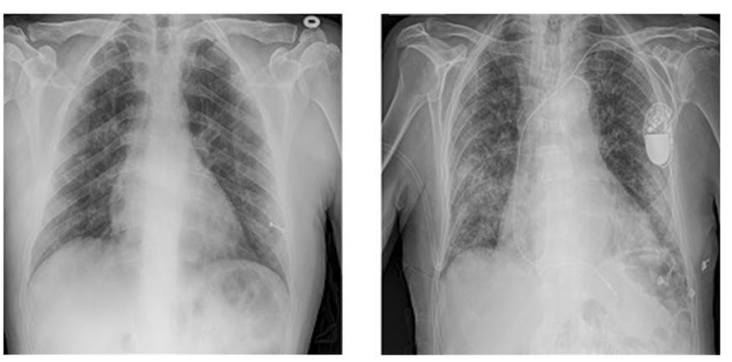

(a) other during training. The generator is trained to produce data similar to the original data and the discriminator should be able to distinguish between fake and actual data.

According to [30], $\mathrm{x}$ be the input data. For learning the generator's distribution $p_{g}$, a prior is defined on input noise variables $p_{z}(z) . \mathrm{G}\left(\mathrm{z} ; \theta_{g}\right)$, defines the data space mapping, where $\mathrm{G}$ corresponds to differentiable function having network parameters $\theta_{g}$. This differentiable function is implemented as a multilayer perceptron network. Also, another multilayer perceptron is define, $\mathrm{D}\left(\mathrm{x} ; \theta_{d}\right)$ that takes the input from $\mathrm{G}$ and produces the final output. $\mathrm{D}(\mathrm{x})$ represents the probability that $\mathrm{x}$ is original or fake. We trained $\mathrm{D}$ to enhance the possibility of assigning the appropriate label to both training instances as well as instances from $\mathrm{G}$. The objective function $V(G, D)$ for both $G$ and $D$ can be combined to train the GAN model as [30]:

$\min _{\mathrm{G}} \max _{\mathrm{D}} \mathrm{V}(\mathrm{G}, \mathrm{D})=\mathrm{E}_{\mathrm{x} \sim \mathrm{p}_{\text {data }(\mathrm{x})}}[\log \mathrm{D}(\mathrm{x})]+\mathrm{E}_{\mathrm{z} \sim \mathrm{p}_{\mathrm{z}}(\mathrm{z})}[\log (1-\mathrm{D}(\mathrm{G}(\mathrm{z})))]$,

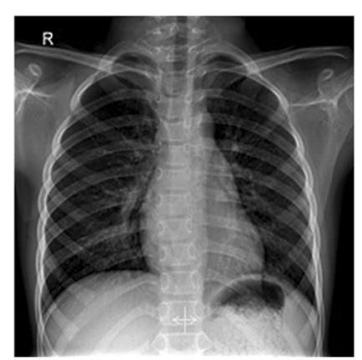

(b)

Fig. 2 Samples of X-ray image dataset used for proposed system; a images of COVID-19 affected cases, $\mathbf{b}$ images of healthy individuals 
Table 2 GAN parameter settings

where $\mathrm{E}$ is the expectation. Equation (1) indicates that we should train the model in such a way that error for $G$ is minimized and D should be maximized so that it should not be able to distinguish between fake and real data.

In this study, the synthetic data were generated using the same architectural setup for both $\mathrm{G}$ and $\mathrm{D}$ as summarized in Table 2. The data augmentation was achieved using horizontal and vertical shifts, and random $\mathrm{r}$.

\subsection{Feature selection}

Feature selection plays a crucial role for raw data representation. It is among top of the list hotest research subject in computer vision and machine learning domain. The main aim is to obtain highly discriminative features from the raw data that have potential to enhance the classification accuracy of the classifier.

The explosion of data set size triggers the development of various data dimensionality reduction techniques to boost the performance of data mining and classification systems. Various feature selection and extraction methods have been developed such as random-forest feature selection, PCA, linear discriminant analysis (LDA), forward feature selection and backward feature elimination methods. We employed PCA as feature extraction technique due to its simplicity, efficiency and popularity for being an oldest multivariate technique.

PCA is a multivariate statistical procedure that analyzes dependent and inter-correlated variables in original dataset and extracts the important information by transforming it to a new set of orthogonal variables called principal components [31]. A new set of principal components is attained, each with certain variance, while the first principal component attains the highest variance among others. Amount of information to retain strongly depends on selection of principal components; therefore, maximum amount of information can be retained by selecting appropriate amount of principal components to reduce data dimensionality.

PCA reduces the 2-D matrix, $\mathrm{X}(\mathrm{N}, \mathrm{M})$ pertaining images, (where $\mathrm{M}$ is total pixels after masking, and $\mathrm{N}$ is number of instances such that $\mathrm{N}<\mathrm{M}$ ), to smaller matrix $\mathrm{Z}(\mathrm{N}, \mathrm{L})$, (where $\mathrm{L}$ is the number of pixels such that $\mathrm{L}<\mathrm{M}$ ), while retaining much information from data, using linear transformation $\mathrm{U}(\mathrm{M}, \mathrm{L})[31,32]$.

$Z=U^{T} X$.

It calculates covariance matrix $\mathrm{S}(\mathrm{L}, \mathrm{L})$ to represent the information as

$S_{Z}=\frac{1}{N} Z^{T} Z$

The maximization of covariance yields eigenvector equations with Lagrange multiplier, $\lambda$. These eigenvector equations are then decomposed using matrix diagonalization that results $\mathrm{S}$ as a product of three matrices:

$S=P D P^{-1}$,

where $D$ corresponds to diagonal matrix, consists of Eigen values, and $\mathrm{P}$ refers to matrix of eigenvector. Therefore, the sum of eigenvalues corresponds to entire variance of the transformation is

Total variance $=\sum_{i=1}^{M} \lambda i$

To project the top L eigenvectors data along the subset of these $\mathrm{M}$ vectors, the variance retained is

Retained variance $=\sum_{i=1}^{L} \lambda i$

Hence, the amount of information retained is expressed as percentage of the original using

Percentage of information retained $=\frac{\sum_{i=1}^{L} \lambda i}{\sum_{i=1}^{M} \lambda i}$

Using these equations, PCA first calculates the mean of every dimension of whole dataset, computes the covariance matrix and determines the eigenvector and eigenvalue pairs using matrix diagonalization. Later, it sorts these pairs by decreasing order of eigenvalues. Since the eigenvalues are proportional to variance retained, the selection of top $L$ pairs will retain most of the information while using only fraction of original dimensions. 


$$
w_{0,0} a_{0}^{(0)}+w_{0.1} a_{1}^{(0)}+\cdots w_{0, n} a_{n}^{(0)}
$$

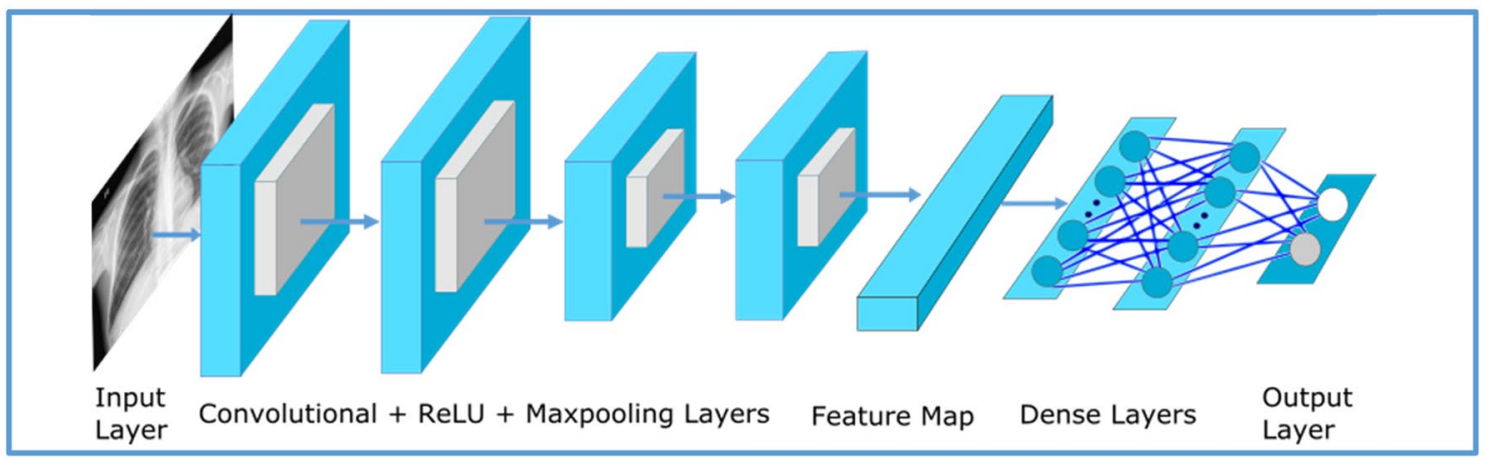

Fig. 3 Architecture of the proposed CNN

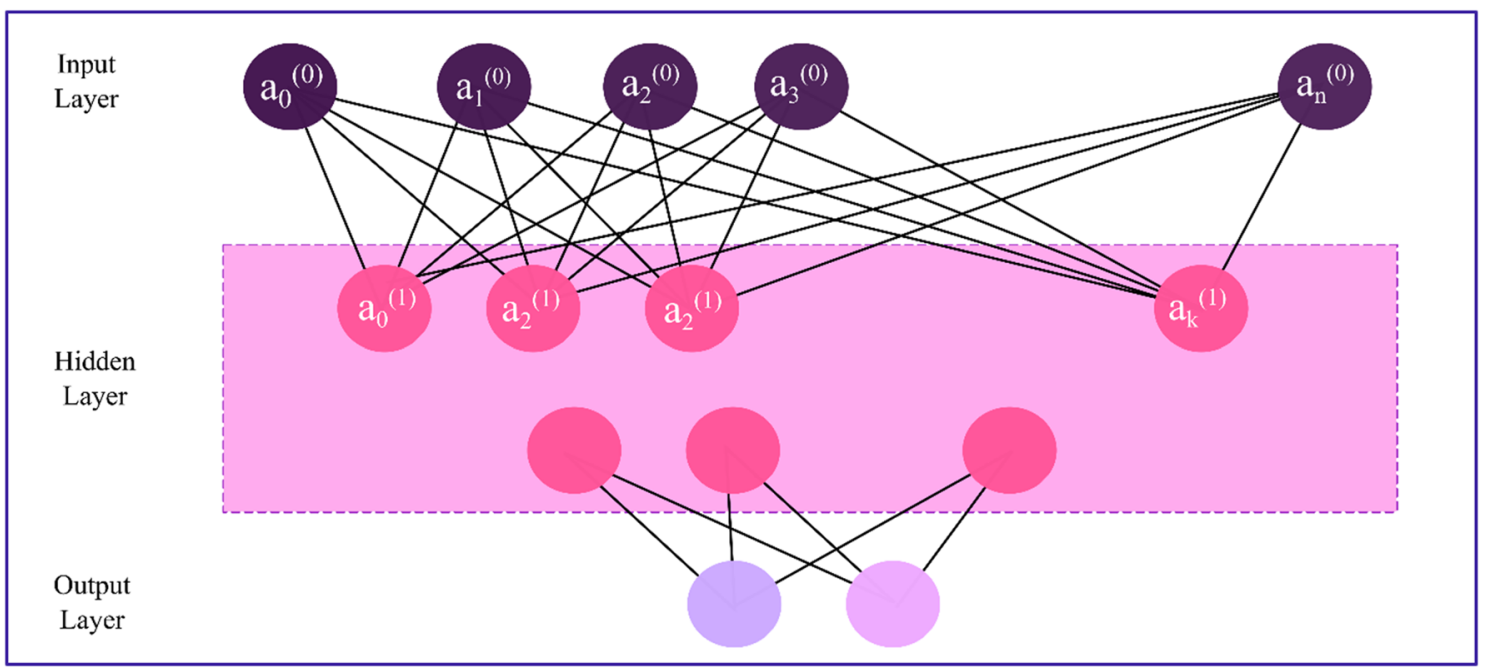

Fig. 4 Visual representation of neurons and weights in CNN

\subsection{Classification methods}

This section describes machine learning and DL models used for this study to identify patients affected by COVID-19 infection. To accomplish the goal of identifying COVID-19 positive patients among normal healthy individuals, CNN and LR models are used, as described in this section.

\subsubsection{Convolutional neural network (CNN)}

$\mathrm{CNN}$, an extensively adopted classification algorithm in DL field, consists an architect of successive layers of perceptron connected in a sequence [33]. It distills the input image data by chain of connected layers and predicts the output by transforming it to meaningful representation. Normally, $\mathrm{CNN}$ architect has three major types of layer; convolutional layer, pooling layer and dense layer or fully connected layer, as depicted in Fig. 3.
Convolutional layer, which is a pivotal layer comprises of neurons connected to small region of preceding layers with some weights of shared characteristics, is shown in hidden layer of Fig. 4. The network takes the image in form of matrix as input map and assigns a weight ' $w$ ' to each one of the connections between neurons of input layer and convolutional layer. It computes weighted sums of all activations ' $a$ ' from the input layer according to these weights:

$w_{0,0} a_{0}^{(0)}+w_{0.1} a_{1}^{(0)}+\ldots w_{0, n} a_{n}^{(0)}$.

As computed weighted-sum might be any number, model normalizes these weighted-sum using functions like $\sigma$ sigmoid that squishes these into range between 0 and 1 . It determines the activation of neurons in layer by adding a suitable bias ' $b$ ' to relevant weighted-sum for meaningful activation. Thus, the first neuron in the second layer is represented by 


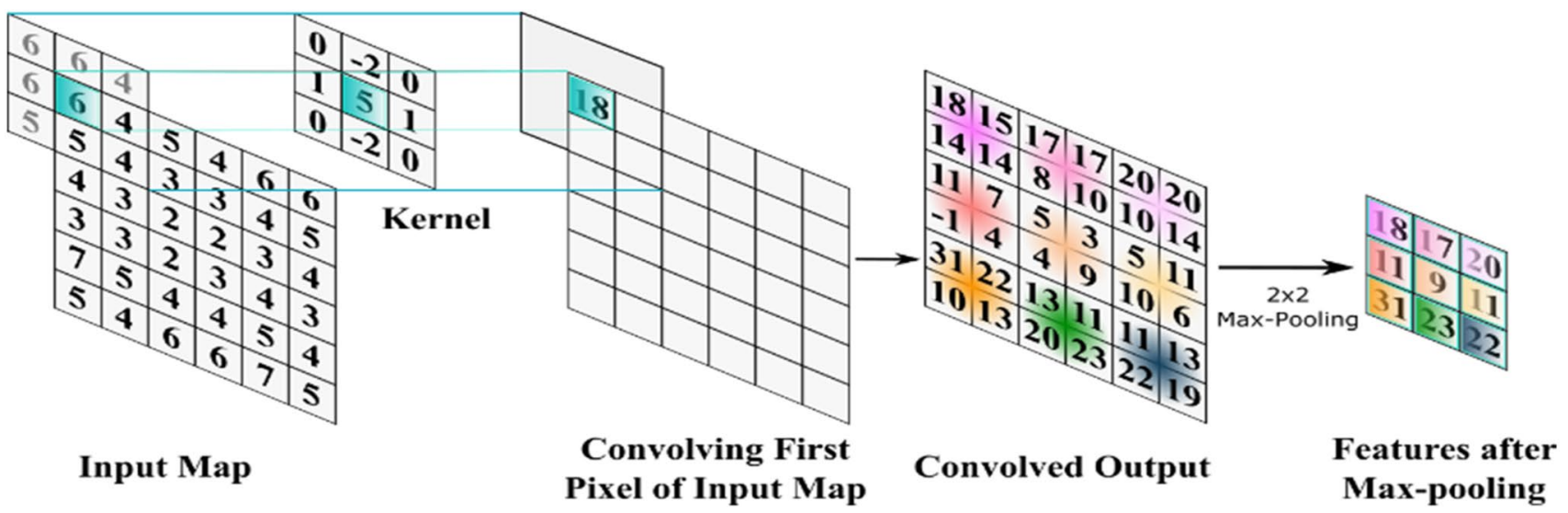

Fig. 5 Visual representation of kernel convolving with input vector and then applying $2 \times 2$ max-pooling

$\left.a_{0}^{1}=\sigma\left(w_{0,0} a_{0}^{(0)}\right)+w_{0}, 1 a_{1}^{0}+\cdots+w_{0, n} a_{n}^{0}+b_{0}\right)$.

The representation of all the neurons can be rearranged in form of matrix that corresponds to activations of next layer.

$\sigma\left(\left[\begin{array}{ccc}w_{0,0} & \cdots & w_{0, n} \\ \vdots & \ddots & \vdots \\ w_{k, 0} & \cdots & w_{k, n}\end{array}\right]\left[\begin{array}{c}a_{0}^{(0)} \\ \vdots \\ a_{n}^{(0)}\end{array}\right]+\left[\begin{array}{c}b_{0} \\ \vdots \\ b_{n}\end{array}\right]\right)=\left[\begin{array}{c}a_{0}^{(1)} \\ \vdots \\ a_{k}^{1}\end{array}\right]$.

Subsequently model discovers right set of weights and biases for all the layers to solve the problem in best way. In convolutional layers, network calculates the scaler product of kernel with input map, as depicted in Fig. 5, to yield activation maps, which are then stacked to perform final output. A non-linear function, rectified linear unit (ReLU), performs elementwise activation with resultant of convolutional layer.

After convolutional layer, pooling layer acts as fuzzy filter that drastically minimizes computational cost by reducing feature dimensionality. Max-pooling and average-pooling are famous among others for feature dimensionality reduction. Max-pooling reduces dimensionality by picking the feature pixel with maximum value among others in the window as illustrated in Fig. 5, whereas average-pooling takes the average of all the pixels in the window.

Finally, a dense layer gathers all the features extracted by previous layers for classification. Later, output layers classifies the input image accordingly by considering the loss based on soft-max layer probabilities.

\subsubsection{Logistic regression (LR)}

LR is a statistical prediction technique in machine learning to perform regression analysis when dependent variable (target) is dichotomous or categorical which can be extended to model of several classes of events [34]. It is named over its core method, known as sigmoid function. This S-shaped curve (shown in Fig. 6) maps real value $\mathrm{x}$ in (11) to values between 0 and 1

$\operatorname{sig}(x)=\frac{1}{1+e^{-x}}$,

where $x$ is the actual input numerical number and $\mathrm{e}$ is the base of natural logarithms.

LR models the probability of predicted output (y) in (12) by combining input $(\mathrm{x})$ with weight and coefficients where $b_{0}$ is bias (intercept term) and $b_{1}$ is coefficient. Maximumlikelihood estimates these beta values from training data to minimize the error in probability prediction.

$y=\frac{e^{b 0+b 1 \times x}}{1+e^{b 0+b 1 \times x}}$.

\section{Experimental results}

Along with the goal of predicting image as positive COVID19 or negative, the primary aim of the research is to enhance the performance of model by performing feature extraction using PCA technique and analyze its effects. To achieve this, the compiled and augmented dataset comprised of 500 images (250 as COVID-19 positive cases and 250 normal healthy individuals) is randomly divided into training subset and testing subset with a ratio of 75:25 respectively. The first set, i.e. training set consists of 375 samples while testing set had 125 instances as depicted in Table 3.

\subsection{Feature extraction using PCA}

To enhance the performance of machine learning models, PCA feature extraction technique is employed. As the compiled X-rays images dataset are of different height and width dimensions, so images were scaled and normalized 


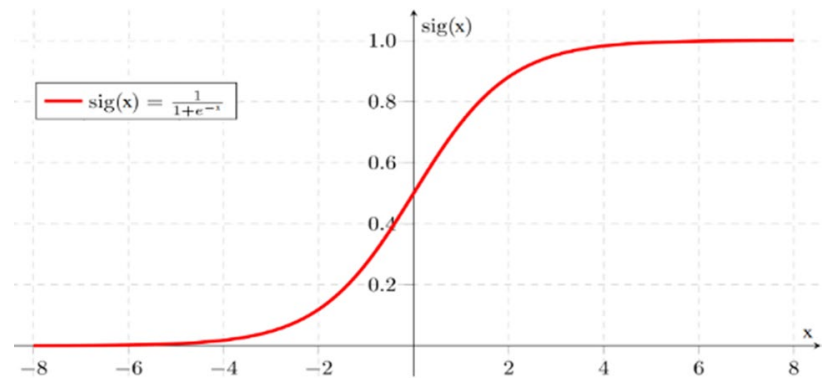

Fig. 6 Core function of LR

Table 3 Dataset split into training and testing set

\begin{tabular}{llll}
\hline Classes & Dataset & Training & Testing \\
\hline COVID-19 cases & 250 & 188 & 62 \\
Normal cases & 250 & 187 & 63 \\
Total & 500 & 375 & 125 \\
\hline
\end{tabular}

as preprocessing step before feeding it to PCA for feature extraction. These normalized images data are then fed to PCA for orthogonal transformation to extract features with the most important data information. Each principal component in new set corresponds to some crucial information as summarized in Table 4. For this experimental setup, only those top features are selected that equates to variance of 1 , $0.99,0.98,0.97,0.96,0.95,0.90$, and 0.85 .

\subsection{Classification}

Once the principal feature sets of dataset are extracted by PCA, each set is then used to train the proposed CNN model and conventional LR network separately. These trained machine learning and DL models are evaluated on respective testing data, and performance analysis is presented in this section.

\subsubsection{CNN}

A CNN model is proposed in Fig. 3 for identification of patients as COVID-19-positive case or a COVID-19-negative case by analyzing an input X-ray image. Respective layers in model transform the input to substantial representation and transmit it to following layers for further operations. The introductory layer in Fig. 3 is the input layer with size of $\mathrm{HxWx} 3$ equivalent to size of input $\mathrm{X}$-ray images. The height ' $H$ ' and width ' $W$ ' of input layer varies in each model according to each principal component feature sets. For feature set with variance $1, \mathrm{H}=\mathrm{W}=512$.

Next to input layer, two convolutional layers are created successively with an output shape of $\mathrm{HxWx} 32$, having a $3 \times 3$ kernel, along with padding of same size to assure the size of spatial output. Afterwards, a $2 \times 2$ max-pool layer reduces features dimensionality, and dropout is fixed to 0.25 . Later, two more convolutional layers are placed with depth of 64 and padding is turned on. A max-pool layer of size $2 \times 2$ takes the outcome of these convolutional layers for dimensionality reduction, and dropout of 0.4 is performed. In all the convolutional layers, ReLU is used as activation function. The resultant features are flattened and passed to dense layer of 128 depth, followed by a dropout of 0.5 . A fully connected layer puts together all the features of preceding layers and the final output of fully connected layer is normalized by shaping probability outcomes into final prediction with a Softmax activation function. The various sets of extracted features are then used to train this $\mathrm{CNN}$ model with Adam optimizer having learning rate of 0.001 , beta_1

Table 4 Pixel values of complete dataset. (A) Normalized pixel value of original dataset images, (B) extracted feature values of top 147 principal components after applying PCA with variance 0.99

\begin{tabular}{|c|c|c|c|c|c|c|c|c|}
\hline \multirow[t]{2}{*}{ Sr. No } & \multicolumn{8}{|c|}{ Original dataset normalized pixel values } \\
\hline & Pixel 0 & Pixel 1 & Pixel 2 & Pixel 3 & $\ldots$ & Pixel 786429 & Pixel 786430 & Pixel 786431 \\
\hline 0 & 0.156863 & 0.156863 & 0.156863 & 0.176471 & $\ldots$ & 0.047059 & 0.047059 & 0.047059 \\
\hline 1 & 0.129412 & 0.129412 & 0.129412 & 0.137255 & $\ldots$ & 0.003922 & 0.011765 & 0.011765 \\
\hline 2 & 0.482353 & 0.482353 & 0.482353 & 0.466667 & $\ldots$ & 0.000000 & 0.000000 & 0.000000 \\
\hline 3 & 0.000000 & 0.000000 & 0.000000 & $\begin{array}{l}0.019608 \\
(\mathrm{~A})\end{array}$ & $\ldots$ & 0.062745 & 0.290196 & 0.290196 \\
\hline \multirow[t]{2}{*}{ Sr. No } & \multicolumn{8}{|c|}{ 147-Principal component values } \\
\hline & PC 1 & PC 2 & PC 3 & PC 4 & $\ldots$ & PC 145 & PC 146 & PC 147 \\
\hline 0 & -27.311999 & -36.931316 & 11.087628 & 22.476118 & $\ldots$ & -3.152277 & 2.403450 & -1.161468 \\
\hline 1 & -72.68627 & 55.72737 & 5.388810 & -3.205570 & $\ldots$ & 0.287251 & -1.940857 & 3.880443 \\
\hline 2 & -30.392400 & -16.253388 & -0.724725 & -18.254980 & $\ldots$ & -1.018936 & 0.819682 & 2.595462 \\
\hline 3 & -7.005396 & -6.036791 & -22.227564 & $\begin{array}{l}-0.651227 \\
\text { (B) }\end{array}$ & $\ldots$ & 0.315235 & 0.417503 & 0.745322 \\
\hline
\end{tabular}


Table 5 Netwok topology of proposed CNN model

\begin{tabular}{|c|c|c|c|c|c|c|c|c|}
\hline \multirow[t]{2}{*}{ Dataset } & \multicolumn{5}{|c|}{ Convolution layers } & \multicolumn{3}{|c|}{ Fully connected layers } \\
\hline & Kernel Size & & ReLU & Max-pooling & Dropout & No. of neurons & Function & Dropout \\
\hline \multirow[t]{5}{*}{ Covid-19 } & $3 \times 3 \times 32$ & & Yes & No & No & & & \\
\hline & $3 \times 3 \times 32$ & & Yes & $2 \times 2$ & Yes $(25 \%)$ & & & \\
\hline & $5 \times 5 \times 64$ & & Yes & No & No & & & \\
\hline & $5 \times 5 \times 64$ & & Yes & $2 \times 2$ & Yes $(40 \%)$ & 128 & ReLU & Yes $(50 \%)$ \\
\hline & & & & & & 2 & Softmax & No \\
\hline \multicolumn{9}{|c|}{ Other parameters } \\
\hline Batch size & & Steps (iterations) & Epochs & Learning rate & Optimizer & & & \\
\hline 8 & & 100 & 25 & 0.001 & Adam & & & \\
\hline
\end{tabular}

of 0.9 , beta_2 of 0.999 while epsilon is set to none and amsgrad to false. CNN network is trained with online augmentation (augmentation on the fly technique) over 25 epochs with a batch size of 8 . Table 5 summarizes the network topology and parameters used in proposed CNN model. Fig. 7 shows the accuracy and loss curves of model against various principal components sets, while the evaluation performance of proposed CNN classification network with different principal components are summarized in Table 6.

\subsubsection{Linear regression}

Beside DL-based CNN model, the extracted features are also fed to conventional LR model for training and testing. All the sets consisting of principal components of various variances are fed to separate LR networks and performance is recorded for each LR model against each set, which is depicted in receiver operating characteristics (ROC) curves, shown in Fig. 8. Table 7 summarizes the classification performance of LR model over selection of various PCA components.

\subsection{Performance analysis}

The classification accuracies of trained models (CNN and LF) corresponding to different PCA sets are summarized in Table 8 along with the time taken by model for training. The experimental results show that without feature extraction, the proposed CNN and LR models achieved accuracy of $97.6 \%$ and $95.2 \%$, respectively. It is prominent by results in Table 8 that proposed CNN framework, trained with dataset of extracted features having 0.99 variance, outperformed other trained models by achieving $100 \%$ accuracy with just $233 \mathrm{~ms}$ of training. CNN model trained on dataset of 0.99 variance, predicted all the testing data correctly, while other models misclassified either a healthy person as COVID-19-infected patient or a COVID-19-infected patient as healthy person (see Fig. 9).

All the experimental work is carried out using Python 3.6 and Keras packages on Jupyter Notebook (version
6.0.3) having $32 \mathrm{~GB}$ system RAM with Intel® processor (Xeon® CPU E3-1231 3.40 GHz). Additionally, the code is implemented with help of graphical processing unit (GPU) NVIDIA Quadro K500.

\section{Summary and discussion}

The clinical and radiological COVID-19 images complied by Dr. Joseph P. Cohen are used by research community to develop models for accurate identification of patients affected with COVID-19 infectious disease. Table 9 summarizes the attempts made by researchers to identify the patients affected by COVID-19 using combinations of various artificial intelligence methods. Togacar et al. [35] introduced DL frameworks based on different CNN architectures that uses X-ray images to diagnose COVID-19. They combined SVM classifier with MobileNetV2 and SqueezeNet, and exploited social mimic optimization method while incorporating fuzzy color scheme with stacking approaches. $99.27 \%$ accuracy and $98.58 \% \mathrm{~F} 1$ score was attained by the researchers on an X-ray images dataset comprising of 295 of COVID-19 positive cases and 163 of healthy or other pneumonia affected patients.

Tuncer et al. [36], with the help of residual exempler local binary pattern (ResExLBP), diagnosed corona virus disease in digital lungs X-ray images by extracting the features, drew on iterative relief (iRF) and fed the resultant to six different machine learning classifiers. The dataset used for the study consists of $87 \mathrm{X}$-ray images related to patients affected by COVID-19 while 234 X-ray images of normal patients. They achieved a maximum accuracy of $99.69 \%$ with an overall sensitivity of $98.85 \%$.

A combination of transfer learning with various $\mathrm{CNN}$ frameworks is adopted by Apostolopoulos and Mpesiana [37] to facilitate the detection of this pandemic disease. They inspected 224 confirmed coronavirus disease, 714 bacterial pneumonia, and 504 ordinary X-ray images and observed that MobileNetV2 outperformed other frameworks by 

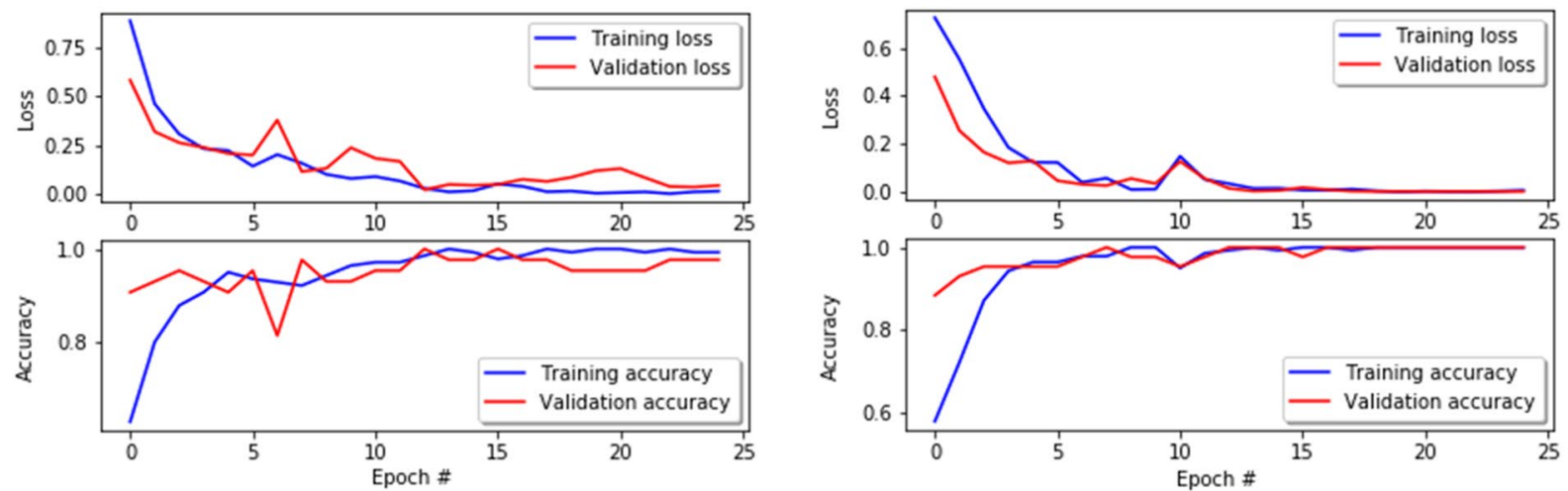

(a)
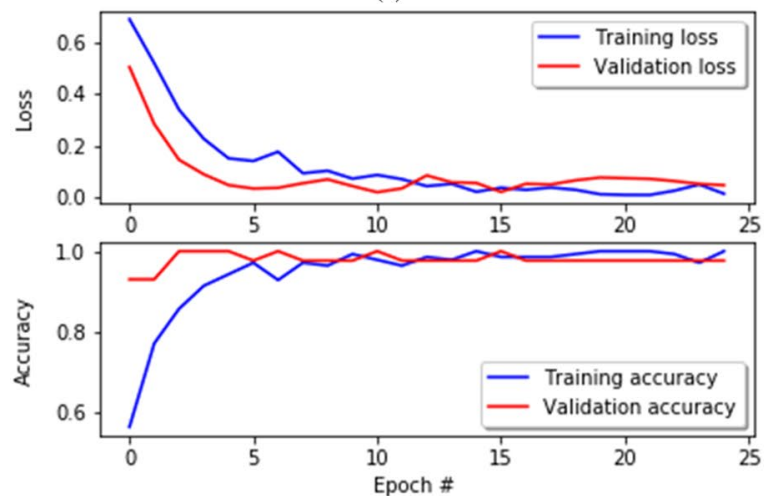

(c)
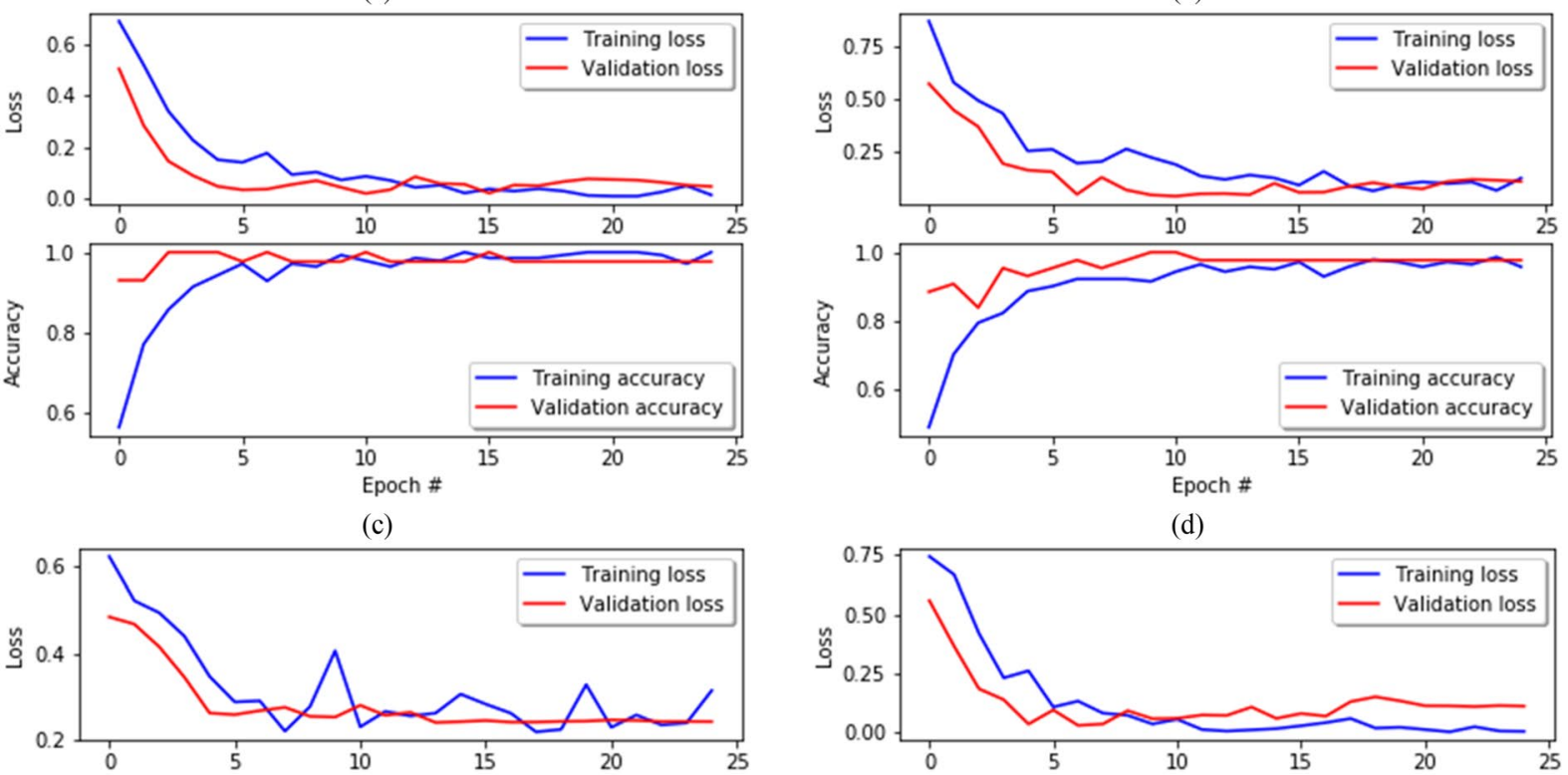

(d)
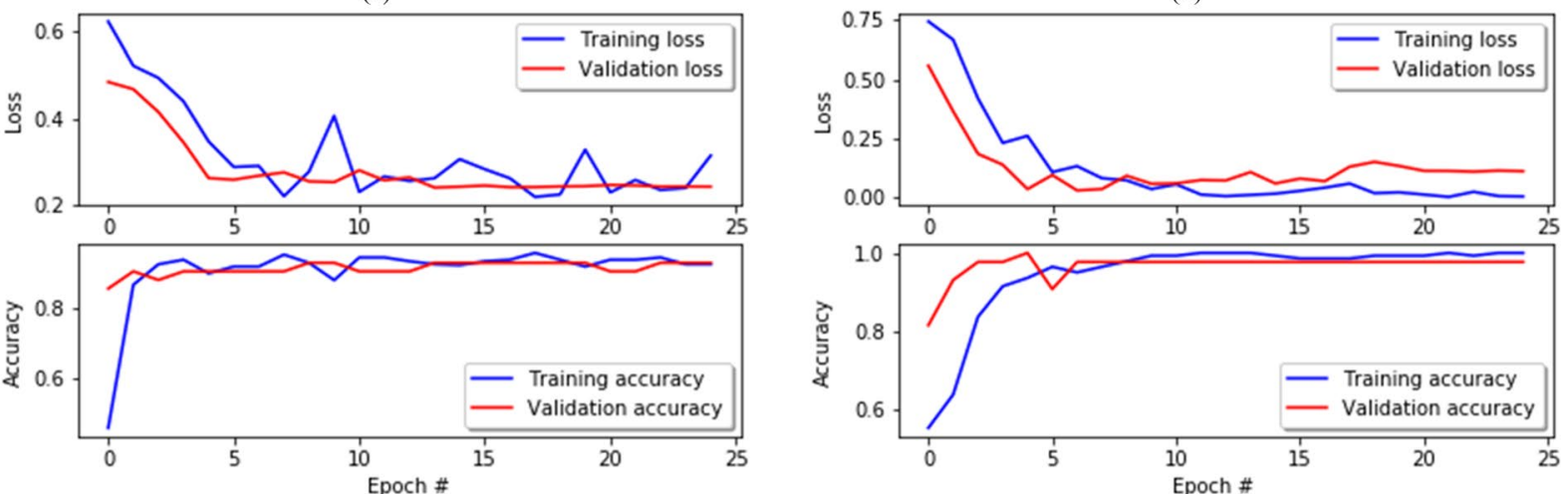

(e)

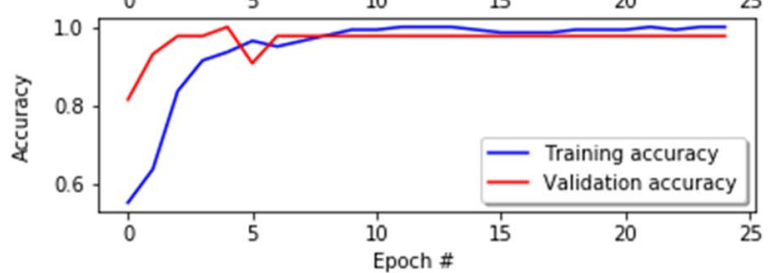

(f)
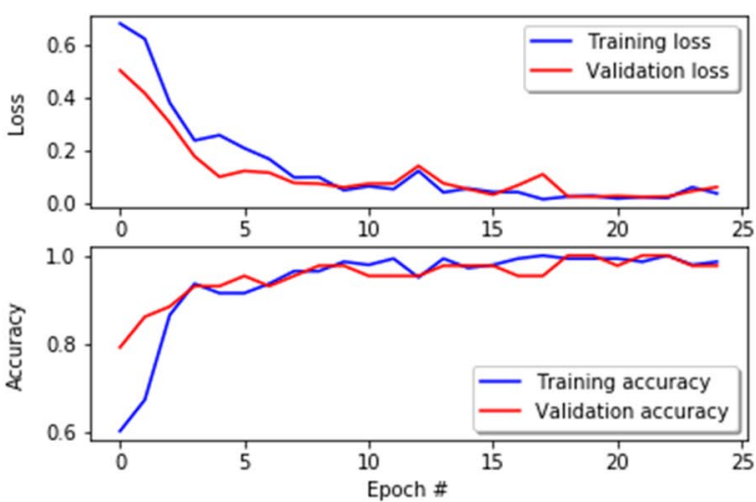

(g)

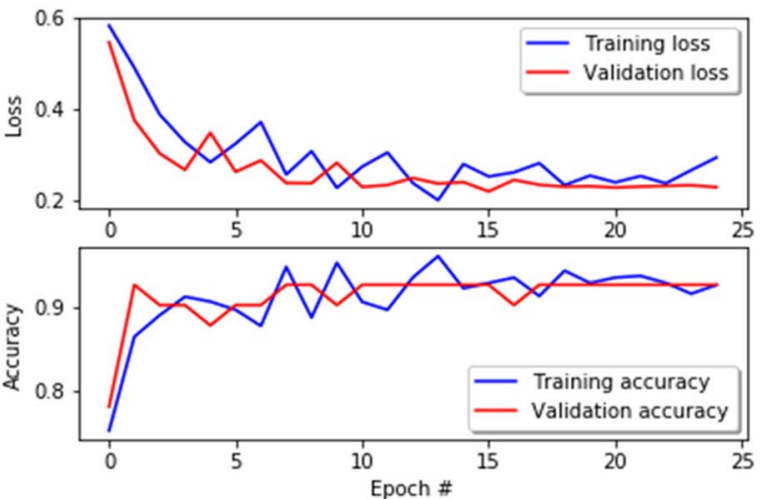

(h) 
4Fig. 7 Training and testing loss/accuracy graphs of CNN network when a variance $=1, \mathbf{b}$ variance $=0.99$ and components $=147, \mathbf{c}$ variance $=0.98$ and components $=126$, $\mathbf{d}$ variance $=0.97$ and components $=108$, e variance $=0.96$ and components $=96, \mathbf{f}$ variance $=0.95$ and components $=84, \mathbf{g}$ variance $=0.90$ and components $=48, \mathbf{h}$ when variance $=0.85$ and components $=30$

securing an accuracy and sensitivity of $97.40 \%$, and $99.10 \%$ respectively. Brunese et al. [38] tailored the transfer learning technique with fine-tuned visual geometry group (VGG-16) model that examined 6523 chest X-rays (250 images of patients affected by COVID-19, 2753 images corresponds to pulmonary diseases patients and 3520 images related to healthy persons). It first segregated between pulmonary diseases patients and healthy person, then classified the X-ray image of patient with former disease either as coronavirus disease positive or other pneumonia-affected person. Thus accomplished a success rate of $97.0 \%$ and $\mathrm{F} 1$ score of $92.0 \%$.

Jaiswal et al. [39] blended DL with deep transfer learning approach by employing a modified pre-trained DenseNet201
Table 6 Performance evaluation of CNN model

\begin{tabular}{|c|c|c|c|c|c|c|c|c|c|}
\hline \multirow{3}{*}{$\begin{array}{l}\text { Variance } \\
\text { Retained }\end{array}$} & \multicolumn{9}{|l|}{$\mathrm{CNN}$} \\
\hline & \multicolumn{3}{|c|}{ Precision } & \multicolumn{3}{|l|}{ Recall } & \multicolumn{3}{|c|}{ F measure } \\
\hline & Corona & Normal & Overall & Corona & Normal & Overall & Corona & Normal & Overall \\
\hline 1 & 0.952 & 1 & 0.976 & 1 & 0.955 & 0.977 & 0.975 & 0.977 & 0.976 \\
\hline 0.99 & 1 & 1 & 1 & 1 & 1 & 1 & 1 & 1 & 1 \\
\hline 0.98 & 0.952 & 1 & 0.976 & 1 & 0.955 & 0.977 & 0.975 & 0.977 & 0.976 \\
\hline 0.97 & 0.952 & 1 & 0.976 & 1 & 0.955 & 0.977 & 0.975 & 0.977 & 0.976 \\
\hline 0.96 & 0.952 & 1 & 0.976 & 1 & 0.955 & 0.977 & 0.975 & 0.977 & 0.976 \\
\hline 0.95 & 0.952 & 1 & 0.976 & 1 & 0.955 & 0.977 & 0.975 & 0.977 & 0.976 \\
\hline 0.90 & 0.952 & 1 & 0.976 & 1 & 0.955 & 0.977 & 0.975 & 0.977 & 0.976 \\
\hline 0.85 & 0.910 & 1 & 0.955 & 1 & 0.913 & 0.957 & 0.952 & 0.955 & 0.945 \\
\hline
\end{tabular}

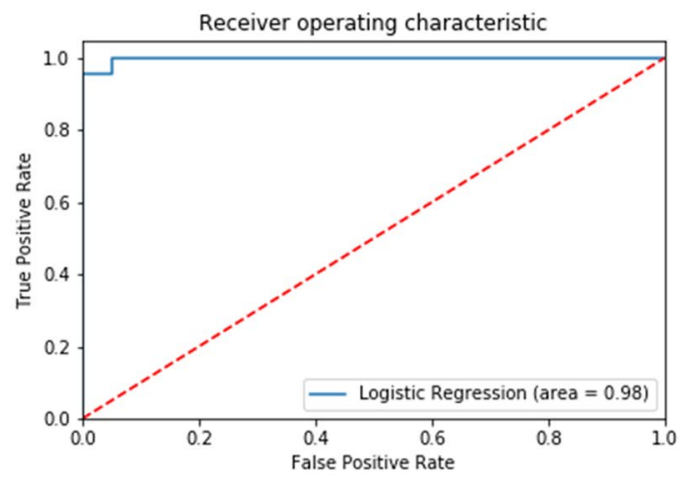

(b)

(a)

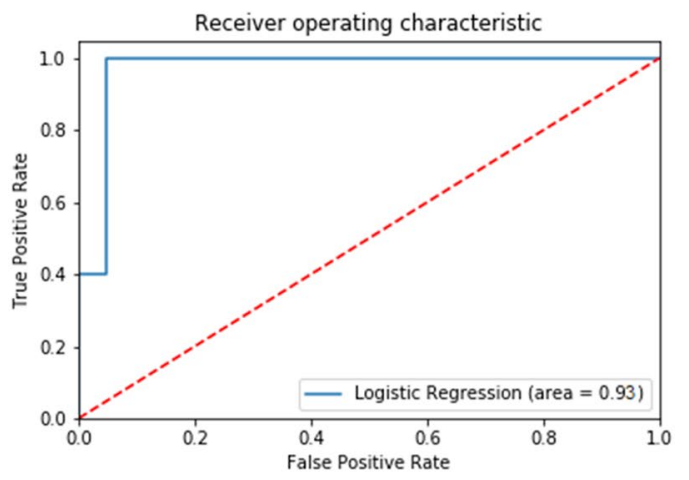

(c)

Fig. 8 ROC curves for logistic regression model when $\mathbf{a}$ variance $=1,0.99,0.98,0.96,0.95$ and $0.90, \mathbf{b}$ variance $=0.97, \mathbf{c}$ variance $=0.85$ 
Table 7 Performance evaluation of linear regression model

\begin{tabular}{|c|c|c|c|c|c|c|c|c|c|}
\hline \multirow{3}{*}{$\begin{array}{l}\text { Variance } \\
\text { retained }\end{array}$} & \multicolumn{9}{|l|}{ LR } \\
\hline & \multicolumn{3}{|c|}{ Precision } & \multicolumn{3}{|l|}{ Recall } & \multicolumn{3}{|c|}{ F-measure } \\
\hline & Corona & Normal & Overall & Corona & Normal & Overall & Corona & Normal & Overall \\
\hline 1 & 0.91 & 1 & 0.955 & 1 & 0.913 & 0.957 & 0.952 & 0.955 & 0.945 \\
\hline 0.99 & 0.91 & 1 & 0.955 & 1 & 0.913 & 0.957 & 0.952 & 0.955 & 0.945 \\
\hline 0.98 & 0.91 & 1 & 0.955 & 1 & 0.913 & 0.957 & 0.952 & 0.955 & 0.945 \\
\hline 0.97 & 0.95 & 1 & 0.976 & 1 & 0.955 & 0.977 & 0.975 & 0.977 & 0.976 \\
\hline 0.96 & 0.91 & 1 & 0.955 & 1 & 0.913 & 0.957 & 0.952 & 0.955 & 0.945 \\
\hline 0.95 & 0.91 & 1 & 0.955 & 1 & 0.913 & 0.957 & 0.952 & 0.955 & 0.945 \\
\hline 0.90 & 0.91 & 1 & 0.955 & 1 & 0.913 & 0.957 & 0.952 & 0.955 & 0.945 \\
\hline 0.85 & 0.90 & 0.95 & 0.930 & 0.95 & 0.913 & 0.932 & 0.927 & 0.933 & 0.930 \\
\hline
\end{tabular}

Table 8 Classification accuracy and computational time to fit proposed models after PCA with different fractions of variance retained

\begin{tabular}{|c|c|c|c|c|c|}
\hline \multirow{2}{*}{$\begin{array}{l}\text { Variance } \\
\text { retained }\end{array}$} & \multirow[t]{2}{*}{ No. of components } & \multicolumn{2}{|c|}{ Time (ms) } & \multicolumn{2}{|c|}{ Accuracy (\%) } \\
\hline & & CNN & LR & $\mathrm{CNN}$ & LR \\
\hline 1 & 786432 & 858000 & 17600 & 97.6 & 95.2 \\
\hline 0.99 & 147 & 233 & 1527 & 100 & 95.2 \\
\hline 0.98 & 126 & 230 & 1521 & 97.6 & 95.2 \\
\hline 0.97 & 108 & 217 & 1519 & 97.6 & 97.6 \\
\hline 0.96 & 96 & 219 & 1508 & 97.6 & 95.2 \\
\hline 0.95 & 84 & 216 & 1508 & 97.6 & 95.2 \\
\hline 0.90 & 48 & 211 & 1499 & 97.6 & 95.2 \\
\hline 0.85 & 30 & 211 & 1493 & 95.2 & 92.8 \\
\hline
\end{tabular}

model for COVID-19 screening among CT images. They used $1262 \mathrm{CT}$ images of COVID-19-positive cases and $1230 \mathrm{CT}$ images of normal patients to secure an accuracy of $96.25 \%$ while F1 score of $96.29 \%$ with addition of data augmentation technique. Similarly, Sharma [40] designed a customized CNN-based ResNet50 architect as an automated tool for coronavirus disease diagnosis using 2200 computed tomography images of lungs ( 800 belongs to COVID-19 positive while 1400 of other pneumonia and normal patients) that attained $91.0 \%$ accuracy with sensitivity of $92.1 \%$.
A SqueezeNet demonstrated by Ucar and Korkmaz [41] is tuned with Bayesian optimization additive for COVID-19 diagnosis. They gained an overall accuracy of $98.30 \%$ on dataset of 5949 chest radiography images that consists of 76 COVID-19 infected cases. Bai et al. [42] incorporated long short term memory (LSTM) with multi-layer perceptron (MLP) for classification of COVID-19 disease. They used 133 samples for training the proposed model and achieved $89.10 \%$ performance rate.

In this research, researchers analyzed the effect of PCA on proposed DL-based CNN model and LR network for detection of COVID-19 in X-ray images. For experimental work, $500 \mathrm{X}$-ray images are used, among which 250 images corresponds to patients affected by COVID-19 and 250 belongs to normal patients. By practicing PCA as feature extraction technique with proposed CNN model, we accomplished $100 \%$ accuracy with less computational time. While same extracted features when fed to LR network, model secured an accuracy of 97.6\%. Evidently, our proposed method $(\mathrm{PCA}+\mathrm{CNN})$ outperformed other studies mentioned in Table 9.

Due to limited COVID-19 chest radiography images available publicly, which is constantly updating, most of the studies mentioned above either used 25,50 or around 100 images related to COVID-19-affected patients in their

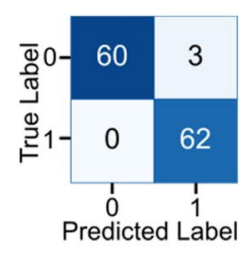

(a)

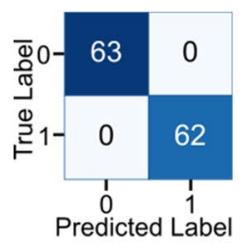

(b)

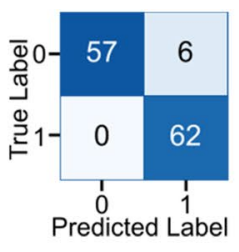

(c)

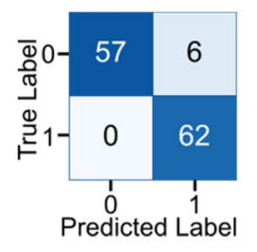

(d)

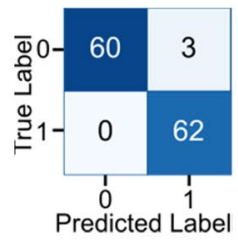

(e)

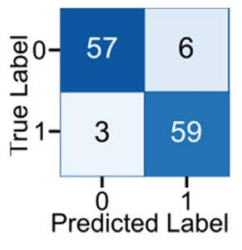

(f)

Fig. 9 Confusion matrix for a CNN model at variance of 1, 0.98, 0.97, 0.96, 0.95, and 0.90, b CNN model at variance of 0.99 , $\mathbf{c}$ CNN model at variance of $0.85, \mathbf{d}$ LR model at variance of $1,0.99,0.98,0.96,0.95$ and 0.90 , e LR model at variance of 0.97 , $\mathbf{f}$ LR model at variance of 0.85 
Table 9 Comparison of current AI diagnostic techniques with proposed methods using different medical radiology images

\begin{tabular}{|c|c|c|c|c|c|c|}
\hline Ref. & Design/technique title & Images Type & Accuracy & Precision & Sensitivity & F1-score \\
\hline [35] & $\begin{array}{l}\text { DL frameworks (SqueezeNet, and MobileNetV2) with additional } \\
\text { SMO method and Support Vector Machine classifier }\end{array}$ & X-ray & 99.2 & 98.8 & 98.3 & 98.5 \\
\hline [36] & Five various ML-based classifiers with iRF feature extraction & $\mathrm{X}$-ray & 99.6 & - & 98.8 & - \\
\hline [37] & $\begin{array}{l}\text { Pre-trained DL-based frameworks (Inception ResNetV2, Inception, } \\
\text { VGG19, Xception, and MobileNet v2) }\end{array}$ & X-ray & 97.4 & - & 99.1 & - \\
\hline [38] & VGG-16 & X-ray & 97.0 & - & 92.0 & 92.0 \\
\hline [39] & Deep CNN with transfer learning based DenseNet201 & $\mathrm{CT}$ & 96.2 & 96.2 & 96.2 & 96.2 \\
\hline [40] & Customized CNN-based ResNet50 & CT & 91.0 & - & 92.1 & - \\
\hline [41] & Deep Bayes-SqueezeNet & X-ray & 98.3 & - & - & 98.3 \\
\hline [43] & Convolutional CAPSNET & X-ray & 97.2 & 97.0 & 97.4 & 97.2 \\
\hline [44] & CSSA-EfficientNet-B0 based on deep learning 2D curvelet transform & X-ray & 99.6 & 99.6 & 99.4 & 99.5 \\
\hline Proposed & $\mathrm{PCA}+\mathrm{CNN}$ & $\mathrm{X}$-ray & 100 & 100 & 100 & 100 \\
\hline
\end{tabular}

dataset for developing artificial intelligence-based classification system. However, in this proposed study, we used 198 images of COVID-19-positive cases, and employed GAN data augmentation technique to avoid overfitting. At large, studies conducted prior to this study generally used ResNet50 as classification technique without implementing any feature extraction method, while this study is based on PCA as feature extraction technique that drastically reduces the computational time as well as enhances the performance of proposed CNN model.

\section{Conclusion}

The study inspected the implementation of machine learning methods for identification of patients affected by COVID-19 with the help of X-ray images. Suggested approach is especially useful to quickly identify the patients so that necessary medical care can be provided in timely manner. In addition, it provides an alternative cheap solution for the situation in developing countries where testing kits are not available or expensive to conduct standard testing at mass scale. Data augmentation technique using GAN was also employed for increasing the amount of the data and to maximize the classification accuracy of proposed classifiers by reducing the risk of overfitting. The main advantage of the DL method was to reduce the do-it-yourself characteristics which are painstaking as well as labor-intensive, thus it improved the classification accuracy based on data-driven feature learning approach. The proposed method achieved high accuracy of $100 \%$ using CNN + PCA when variance of 0.99 was used. Moreover, the training time was also drastically reduced with the incorporation of PCA while achieving 100\% accuracy on testing data. The proposed $\mathrm{CNN}+\mathrm{PCA}$ technique eclipsed the ultra-modern and advanced approaches as it attained highest accuracy.
The researchers of this study plan to coach the network on relatively substantial dataset and then apply the network to improve the reliability of decision and overall accuracy. Moreover, the architecture of the CNN network can be made more complex by adding additional layers, for larger data set, to enhance the system capabilities of learning highly abstract features, thus making it less prone to overfitting. In addition to the image data, other features such as body temperature, information about presence of chronic diseases such as diabetes, heart etc. will be integrated with the system to make it more robust and reliable to support healthcare actioners. The proposed work can also be adopted for other medical applications such as breast cancer detection, tumor detection etc.

Funding The authors declare that they have no known competing financial interests or personal relationships that could have appeared to influence the work reported in this paper.

\section{Compliance with ethical standards}

Conflict of interest The authors declare that they have no conflict of interest.

Informed consent For this type of study, formal consent is not required.

Human and animal rights This paper does not contain any studies with human participants or animals performed by any of the authors.

\section{References}

1. Tyrrell DA, Bynoe M (1966) Cultivation of viruses from a high proportion of patients with colds. Lancet 287:76-77. https://doi. org/10.1016/S0140-6736(66)92364-6

2. Kahn JS, McIntosh K (2005) History and recent advances in coronavirus discovery. Pediatr Infect Dis J 24:S223-S227. https://doi. org/10.1097/01.inf.0000188166.17324.60 
3. Jain V, Yuan J-M (2020) Predictive symptoms and comorbidities for severe COVID-19 and intensive care unit admission: a systematic review and meta-analysis. Int J Public Health 65:533-546. https://doi.org/10.1007/s00038-020-01390-7

4. Ren Y, Li L, Jia Y (2020) New method to reduce COVID-19 transmission: the need for medical air disinfection is now. J Med Syst 44:119. https://doi.org/10.1007/s10916-020-01585-8

5. Fisher D, Heymann D (2020) Q and A: the novel coronavirus outbreak causing COVID-19. BMC Med 18:57. https://doi. org/10.1186/s12916-020-01533-w

6. Seshadri DR, Davies EV, Harlow ER, Hsu JJ, Knighton SC, Walker TA, Voos JE, Drummond CK (2020) Wearable sensors for COVID-19: a call to action to harness our digital infrastructure for remote patient monitoring and virtual assessments. Front Digit Health 2:8. https://doi.org/10.3389/fdgth .2020 .00008

7. Yahyaoui A, Jamil A, Rasheed J, Yesiltepe M (2019) A decision support system for diabetes prediction using machine learning and deep learning techniques. In 2019 1st international informatics and software engineering conference (UBMYK). IEEE. https://doi.org/10.1109/UBMYK48245.2019.8965556

8. Nallamala SH, Mishra P, Koneru SV (2019) Breast cancer detection using machine learning way. Int J Recent Technol Eng 8:1402-1405

9. Gurbina M, Lascu M, Lascu D (2019) Tumor detection and classification of MRI brain image using different wavelet transforms and support vector machines. 2019 42nd international conference on telecommunications and signal processing. TSP. https ://doi.org/10.1109/TSP.2019.8769040

10. Sun P, Wang D, Mok VC, Shi L (2019) Comparison of feature selection methods and machine learning classifiers for radiomics analysis in glioma grading. IEEE Access 7:102010-102020. https://doi.org/10.1109/access.2019.2928975

11. de Bruijne M (2016) Machine learning approaches in medical image analysis: From detection to diagnosis. Med Image Anal 33:94-97. https://doi.org/10.1016/j.media.2016.06.032

12. Nanni L, Ghidoni S, Brahnam S (2017) Handcrafted vs non-handcrafted features for computer vision classification. Pattern Recognit 71:158-172. https://doi.org/10.1016/j.patcog.2017.05.025

13. Ayan E, Ünver HM (2019) Diagnosis of pneumonia from chest $\mathrm{X}$-ray images using deep learning. 2019 scientific meeting on electrical-electronics and biomedical engineering and computer science. EBBT. https://doi.org/10.1109/EBBT.2019.8741582

14. Huynh HT, Anh VNN (2019) A deep learning method for lung segmentation on large size chest x-ray image. RIVF 2019. Proceed IEEE-RIVF Int Conf Comput Commun Technol. https:// doi.org/10.1109/RIVF.2019.8713648

15. Van Tulder G, De Bruijne M (2016) Combining generative and discriminative representation learning for lung CT analysis with convolutional restricted Boltzmann machines. IEEE Trans Med Imaging 35:1262-1272. https://doi.org/10.1109/ TMI.2016.2526687

16. Bardou D, Zhang K, Ahmad SM (2018) Classification of Breast Cancer Based on Histology Images Using Convolutional Neural Networks. IEEE Access 6:24680-24693. https:// doi.org/10.1109/ACCESS.2018.2831280

17. Shin H, Roth HR, Gao M, Lu L, Xu Z, Nogues I, Yao J, Mollura D, Summers RM (2016) Deep convolutional neural networks for computer-aided detection: CNN architectures, dataset characteristics and transfer learning. IEEE Trans Med Imaging 35:12851298. https://doi.org/10.1109/TMI.2016.2528162

18. Zhou X.-Y, Zheng J.-Q, Li P, Yang G.-Z. (2020) ACNN: a Full Resolution DCNN for Medical Image Segmentation. In: 2020 IEEE International Conference on Robotics and Automation (ICRA). IEEE https://doi.org/10.1109/ICRA40945.2020.91973 28.
19. Sudheer Kumar E, Shoba Bindu C (2019) Medical image analysis using deep learning: a systematic literature review. Commu Comput Informat Sci. https://doi.org/10.1007/978-981-13-8300-7_8

20. Russakovsky O, Deng J, Su H, Krause J, Satheesh S, Ma S, Huang Z, Karpathy A, Khosla A, Bernstein M, Berg AC, Fei-Fei L (2015) ImageNet large scale visual recognition challenge. Int J Comput Vision (IJCV) 115:211-252. https://doi.org/10.1007/ s11263-015-0816-y

21. Salehinejad H, Colak E, Dowdell T, Barfett J, Valaee S (2019) Synthesizing chest X-Ray pathology for training deep convolutional neural networks. IEEE Trans Med Imaging 38:1197-1206. https://doi.org/10.1109/TMI.2018.2881415

22. Shorten C, Khoshgoftaar TM (2019) A survey on image data augmentation for deep learning. Journal of Big Data 6:60. https://doi. org/10.1186/s40537-019-0197-0

23. Zhang C, Song Y, Liu S, Lill S, Wang C, Tang Z, You Y, Gao Y, Klistorner A, Barnett M, Cai W (2018) MS-GAN: GAN-based semantic segmentation of multiple sclerosis lesions in brain magnetic resonance imaging. In: 2018digital image computing. Tech Appl (DICTA). https://doi.org/10.1109/DICTA.2018.8615771

24. Oktay O, Ferrante E, Kamnitsas K, Heinrich M, Bai W, Caballero J, Cook SA, de Marvao A, Dawes T, O'Regan DP, Kainz B, Glocker B, Rueckert D (2018) Anatomically constrained neural networks (ACNNs): application to cardiac image enhancement and segmentation. IEEE Trans Med Imaging 37:384-395. https ://doi.org/10.1109/TMI.2017.2743464

25. Schlemper J, Caballero J, Hajnal JV, Price AN, Rueckert D (2018) A deep cascade of convolutional neural networks for dynamic MR image reconstruction. IEEE Trans Med Imaging 37:491-503. https://doi.org/10.1109/TMI.2017.2760978

26. Albarqouni S, Baur C, Achilles F, Belagiannis V, Demirci S, Navab N (2016) AggNet: deep learning from crowds for mitosis detection in breast cancer histology images. IEEE Trans Med Imaging 35:1313-1321. https://doi.org/10.1109/TMI.2016.25281 20

27. Pereira S, Pinto A, Alves V, Silva CA (2016) Brain tumor segmentation using convolutional neural networks in MRI images. IEEE Trans Med Imaging 35:1240-1251. https://doi.org/10.1109/ TMI.2016.2538465

28. Yang X, Fan D, Ren A, Zhao N, Alam M (2019) 5G-based usercentric sensing at C -band. IEEE Trans Industr Inf 15:3040-3047. https://doi.org/10.1109/TII.2019.2891738

29. Yang X, Shah SA, Ren A, Zhao N, Fan D, Hu F, Ur Rehman M, von Deneen KM, Tian J (2018) Wandering pattern sensing at S-band. IEEE J Biomed Health Inform 22:1863-1870. https://doi. org/10.1109/JBHI.2017.2787595

30. Goodfellow I.J, Pouget-Abadie J, Mirza M, Xu B, Warde-Farley D, Ozair S, Courville A, Bengio Y (2014) Generative adversarial nets. In: proceedings of the 27th international conference on neural information processing systems 2, 2672-2680. MIT Press, Cambridge, MA, USA

31. Abdi H, Williams LJ (2010) Principal component analysis. Wiley Interdiscipl Rev Comput Statist 2:433-459. https://doi. org/10.1002/wics.101

32. Kroonenberg PM, de Leeuw J (1980) Principal component analysis of three-mode data by means of alternating least squares algorithms. Psychometrika 45:69-97. https://doi.org/10.1007/BF022 93599

33. Albawi S, Mohammed TA, Al-Zawi S (2017) Understanding of a convolutional neural network. Int Conf Eng Technol (ICET). https ://doi.org/10.1109/ICEngTechnol.2017.8308186

34. Peng C-YJ, Lee KL, Ingersoll GM (2002) An introduction to logistic regression analysis and reporting. J Educ Res 96:3-14. https:// doi.org/10.1080/00220670209598786

35. Toğaçar M, Ergen B, Cömert Z (2020) COVID-19 detection using deep learning models to exploit social mimic optimization and 
structured chest X-ray images using fuzzy color and stacking approaches. Comput Biol Med. https://doi.org/10.1016/j.compb iomed.2020.103805

36. Tuncer T, Dogan S, Ozyurt F (2020) An automated residual exemplar local binary pattern and iterative relief based corona detection method using lung X-ray image. Chemomet Intell Lab Syst 203:104054. https://doi.org/10.1016/j.chemolab.2020.104054

37. Apostolopoulos ID, Mpesiana TA (2020) Covid-19: automatic detection from X-ray images utilizing transfer learning with convolutional neural networks. Phys Eng Sci Med 43:635-640. https ://doi.org/10.1007/s13246-020-00865-4

38. Brunese L, Mercaldo F, Reginelli A, Santone A (2020) Explainable deep learning for pulmonary disease and coronavirus COVID19 detection from X-rays. Comput Methods Programs Biomed 196:105608. https://doi.org/10.1016/j.cmpb.2020.105608

39. Jaiswal A, Gianchandani N, Singh D, Kumar V, Kaur M (2020) Classification of the COVID-19 infected patients using DenseNet201 based deep transfer learning. J Biomol Struct Dyn. https://doi.org/10.1080/07391102.2020.1788642

40. Sharma S (2020) Drawing insights from COVID-19-infected patients using CT scan images and machine learning techniques: a study on 200 patients. Environ Sci Pollut Res. https://doi. org/10.1007/s11356-020-10133-3

41. Ucar F, Korkmaz D (2020) COVIDiagnosis-Net: deep bayesSqueezeNet based diagnosis of the coronavirus disease 2019 (COVID-19) from X-ray images. Med Hypotheses 140:109761. https://doi.org/10.1016/j.mehy.2020.109761

42. Bai X, Fang C, Zhou Y, Bai S, Liu Z, Xia L, Chen Q, Xu Y, Xia T, Gong S, Xie X, Song D, Du R, Zhou C, Chen C, Nie D, Qin L, Chen W (2020) Predicting COVID-19 malignant progression with AI techniques. SSRN Elect J. https://doi.org/10.2139/ssrn.35579 84

43. Toraman S, Alakus TB, Turkoglu I (2020) Convolutional capsnet: A novel artificial neural network approach to detect COVID19 disease from X-ray images using capsule networks. Chaos Solitons Fractals. 140:110122. https://doi.org/10.1016/j.chaos .2020 .110122

44. Altan A, Karasu S (2020) Recognition of COVID-19 disease from X-ray images by hybrid model consisting of $2 \mathrm{D}$ curvelet transform, chaotic salp swarm algorithm and deep learning technique. Chaos, Solitons Fractals. https://doi.org/10.1016/j.chaos .2020 .110071 